\title{
La teoría del estrés crónico como modelo científico en neurociencia cognitiva
}

\author{
The theory of chronic stress as a scientific model \\ in Cognitive Neuroscience \\ Manolete Moscoso ${ }^{1}$ y Eliana Delgado \\ University of South Florida, TAMpa Bay, USA \\ Universidad Nacional Mayor de San Marcos, Perú
}

(RECiBIDO 15/04/2015, ACEPTADO 20/06/2015)

\begin{abstract}
RESUMEN
El estudio científico del estrés presenta serias limitaciones teóricas y metodológicas, las cuales incluyen restricciones conceptuales relacionadas con el antiguo principio de homeostasis como noción central del proceso de adaptación. Un nuevo modelo de estudio acerca del estrés crónico, alostasis y carga alostática explican las interacciones entre el cerebro y los sistemas nervioso e inmune en la causalidad de enfermedades físicas y mentales. Este nuevo marco metodológico de investigación científica refleja el avance empírico observado en el campo de las neurociencias cognitiva y del comportamiento. El impacto negativo del estrés crónico en la salud del individuo pone énfasis en el rol central que cumple el distrés emocional percibido. Se examina la eficacia y efectos de la terapia de reducción de estrés basada en mindfulness (MBSR) a través del diseño de un cuidadoso estudio piloto como una alternativa de tratamiento en el campo de reducción del estrés crónico en pacientes con cáncer y los familiares responsables de su cuidado.
\end{abstract}

Palabras clave: Estrés crónico, neurociencias, mindfulness, cáncer.

\begin{abstract}
The scientific study of stress presents serious conceptual and methodological limitations. The theory and concepts of allostasis and allostasis load allowed a new integrative framework of the concept of chronic stress, that identifies the brain as the mediator of the interactions among the central nervous system and immune system in the development of disease. We examined the negative impact of chronic stress and the perceived emotional distress on illness. Our research on the Mindfulness Based Stress Reduction (MBSR) serves as an illustration of psychological and neurobiological outcomes; and how this therapeutic approach facilitates the reduction of perceived emotional distress in cancer patients and their caregivers.
\end{abstract}

Keywords: Chronic stress, neuroscience, mindfulness, cancer.

1 Ph.D. University of South Florida / Health Schools of Medicine, Public Health, and Nursing. E-mail: mmoscoso@health. usf.edu. Web: www.manoletemoscoso.com 


\section{LA TEORÍA DEL ESTRÉS CRÓNICO COMO MODELO CIENTÍFICO EN NEUROCIENCIA COGNITIVA}

El estudio del estrés en el siglo XX estuvo caracterizado por la naturaleza ambivalente y etérea del concepto de estrés per se, así como también por la diversidad de connotaciones que ofrece, y su limitada utilidad para lograr explicar científicamente cómo el organismo se adapta a situaciones adversas de la vida diaria (Moscoso, 1995). El concepto de estrés no tiene una valoracion definida y carece de especificidad debido a su naturaleza fenomenológica. Para muchas personas el estrés representa un reto que estimula y motiva la obtención de logros y metas, mientras que en otros casos el estrés representa un estado de preocupación, irritabilidad, temor y frustración (Moscoso, 2010). Al margen de estas interpretaciones, el estrés es una condición inevitable en la existencia humana y predispone al organismo para actuar de manera más efectiva ante las exigencies del ambiente. En ese sentido, el estrés es positivo por cuanto permite aprender y desarrollar un buen nivel de resiliencia en el proceso de adaptación. Nuestra posición científica, basada en evidencia empírica disponible en la actualidad, nos permite indicar que el estrés no es necesariamente una experiencia nociva, siempre y cuando se entienda que la percepción del evento estresor determina lo que es "estresante" (Moscoso, 2011).

La falta de especificidad del concepto de estrés no ha permitido una adecuada medición psicométrica, y, por el contrario, ha generado una notoria debilidad metodológica en esta línea de investigación (Spielberger \& Moscoso, 1996). Esta primera limitación conceptual del fenómeno de estrés es muy importante debido a que en investigación científica es crítico reconocer que la claridad conceptual es esencial para el avance de la ciencia. En este sentido, el nuevo marco conceptual basado en la noción de estrés crónico cumple un rol central dentro del modelo operacional de esta línea de investigación. Un significativo número de laboratorios en las áreas de neurociencia cognitiva y del comportamiento en general, y en nuestro propio laboratorio en la Universidad de South Florida en particular, utiliza este nuevo modelo metodológico que incluye y considera al estrés crónico como una noción muy importante en el continuum de salud y enfermedad.

Una segunda restricción metodológica en el estudio del estrés está relacionada con el antiguo principio de homeostasis como noción central del proceso de adaptación. Este concepto fue inicialmente presentado por Walter Cannon (1915) y descrito con mayor detalle por Hans Selye (1936). El principio de adaptación homeostática fue el tema central en el modelo de estrés descrito por Cannon (1932), en el cual se refiere a la estabilidad de los sistemas fisiológicos que mantienen la vida en estado de homeostasis. Dicho sentido de estabilidad que Cannon le dio al concepto de homeostasis demostró ser un error metodológico en el estudio del fenómeno de estrés y el proceso de adaptación. Contrario a esta antigua noción de "estabilidad fisiológica", los estudios actuales acerca del estrés en las ciencias biomédicas han demostrado que la fisiología de los organismos se caracterizan por su dinamismo y cambio permanente dentro de un continuum de parámetros especificos (McEwen \& Wingfield, 2003). 
Este esfuerzo hacia un nuevo modelo conceptual de investigación científica en las áreas de ciencias biomédicas y comportamentales ha permitido la incorporación de conceptos y variables mediadoras de los aspectos neurobiológicos y cognitivos, los cuales presentan objeciones importantes a la noción tradicional de homeostasis, planteando su reconsideración desde el punto de vista científico (Schulkin, 2003). En este sentido, las observaciones de las interacciones bioquímicas del cerebro, el sistema nervioso central y el sistema inmune, así como también las influencias de los principios cognitivos de appraisal y reacciones emocionales como elementos básicos en el proceso de estrés, han permitido dejar de lado el concepto "estático" de homeostasis; estimulando el desarrollo de un nuevo marco conceptual conocido como alostasis y carga alostática (McEwen \& Stellar, 1993; Sterling \& Eyer, 1988).

El propósito del presente trabajo es describir el marco conceptual del estrés crónico como fundamento científico de un nuevo modelo metodológico y multidisciplinario para el estudio de la salud y enfermedad en el área de neurociencias. Asimismo, nuestra segunda intención es presentar observaciones empíricas de naturaleza cognitiva, somática y neurobiológica en relación con el proceso de adaptación de pacientes oncológicos en nuestros estudios de reducción de estrés basado en mindfulness (MBSR) en la Universidad de South Florida.

\section{El estrés crónico: "el villano de la película"}

El nuevo modelo metodológico del estrés presenta distinciones importantes entre el estrés agudo y el estrés crónico. El estrés crónico tiene especial relevancia en el proceso de adaptación del individuo, rasgos de resiliencia y los mecanismos neurocognitivos relacionados con los conceptos de salud y enfermedad. Esta nueva visión de cronicidad del estrés ha recibido enorme atención en la últimas dos décadas debido a su significativa asociación con enfermedades de los sistemas nervioso, inmune y endocrino (Dallman, 2003). En nuestra labor científica definimos el estrés crónico como el resultado de demandas y presiones durante periodos de tiempo prolongados "percibidos" como eternos o interminables. La prevalencia de este estado emocional es observada en pacientes con cáncer, personas expuestas a situaciones de pobreza, en individuos responsables del cuidado prolongado de personas con discapacidad física y pacientes que sufren de enfermedades crónicas (Moscoso, 2014).

Estudios empíricos en neurociencia cognitiva reportan niveles elevados de estrés crónico y alteraciones neurobiológicas en jóvenes y adultos con experiencias traumáticas en la etapa prenatal y desarrollo temprano (Lupien, McEwen, Gunnar \& Heim, 2009). Dichos estudios indican que el estrés crónico se inicia mayormente en la etapa de gestación. Estas experiencias adversas en una edad temprana tienden a generar una visión hostil y amenazante del mundo, permitiendo el desarrollo de un conjunto de actitudes negativas precursoras de estados depresivos y de ansiedad en la vida adulta (McEwen, 2007). La percepción individual o interpretación del estresor es de naturaleza cognitiva, 
existiendo una base empírica de correlatos neurobiológicos en el lóbulo prefrontal del cerebro. La experiencia de distrés emocional causada por el estrés crónico es un proceso cognitivo que genera un desbalance en la formación de emociones negativas y positivas, inclinando la balanza hacia un incremento de emociones negativas y el consecuente desencadenamiento de cuadros de depresión clínica. Esta desregulación emocional-cognitiva del individuo cumple un rol desencadenante en una serie de mecanismos de activacion neurobiológica del sistema límbico y de manera particular en el lóbulo prefrontal, amígdala e hipocampo (McEwen, 2007).

El modelo cognitivo del estrés crónico presenta una significativa asociación empírica con los procesos bioquímicos en el lóbulo prefrontal del cerebro, por lo cual es importante observar que la experiencia del estrés crónico se inicia en el cerebro, afecta al cerebro, y, a su vez, a los sistemas inmune y endocrino (McEwen, 1998; Sapolsky, 1996). El cerebro es el órgano central de la respuesta neurobiológica del estrés y sus efectos sobre el proceso de salud y enfermedad del individuo. Los estudios publicados en la disciplina de la neurociencia cognitiva demuestran que la respuesta del estrés crónico facilita el inicio de un estado de distrés emocional, en el que se observa una excesiva utilización o manejo ineficiente de hormonas, corticoesteroides y catecolaminas en los ejes neuroendocrinos (McEwen, 2007). Este proceso neurobiológico causado por el estrés crónico produce un impacto negativo en el sistema nervioso, activando cambios bioquímicos y un desbalance hormonal que repercuten en los sistemas inmune y neuroendocrino (Dhabhar \& McEwen, 1997).

El avance en la literatura científica del estrés crónico ha facilitado una comprensión más amplia de la importancia del ambiente social y estilos de vida como factores mediadores del impacto negativo en la salud física y mental del individuo. El impacto negativo propicia el desarrollo de enfermedades metabólicas como la inflamación crónica, la artritis reumatoide, fibromialgia, enfermedades cardiovasculares, hipertensión arterial, diabetes tipo 2, obesidad, cáncer, depresión y enfermedades inmunosupresoras. Este deterioro de la salud se presenta como consecuencia de niveles elevados de distrés emocional y la interacción multidireccional a través de ciertas estructuras cerebrales como, por ejemplo, el lóbulo prefrontal, la corteza cingulada anterior, la amígdala, el hipocampo y los sistemas nervioso, simpático y parasimpático (Taub, 2008). A pesar de que ambos sistemas están organizados para proteger el organismo y lograr un equilibrio alostático ante una situación aguda de estrés, irónicamente el cerebro y los sistemas nervioso central y autónomo contribuyen a crear un desbalance bioquímico durante la experiencia del estrés crónico. 


\section{Efectos del estrés crónico sobre el cerebro y sistema inmune}

Los efectos del estrés crónico en la salud del individuo han sido ampliamente descritos en la literatura médica y psicológica en el último cuarto de siglo (Moscoso, 2010; Moscoso, 2014). Existe suficiente evidencia científica que demuestra la elevada asociación de este fenómeno con un significativo número de enfermedades (MacPherson, Dinkel \& Sapolski, 2005 ). A pesar de este progreso, aún existe confusión en relacion con los mecanismos neurobiológicos que cumplen un rol central y originan un desbalance neurobiológico que causa la pérdida de la salud. En este sentido, consideramos importante plantear dos interrogantes que nos permitan examinar estas dudas de manera empírica: (1) ¿En qué momento y circunstancias la reacción neurobiológica generada por el estrés deja de ser protectora del proceso alostático y adaptativo, para convertirse en un proceso nocivo para la salud y el bienestar general? (2) ¿Cómo es posible que el mismo mecanismo supuesto a proteger y mantener el principio de alostasis inicie un proceso opuesto dirigido a deteriorar la salud del individuo?

Las respuestas a estas dos interrogantes requieren primeramente un claro entendimiento del fenómeno denominado la respuesta neurobiológica del estrés, también conocida como "fight-or-flight response", la cual describiremos brevemente a continuación. La respuesta neurobiológica del estrés, descrita por Selye (1936), permite al organismo reaccionar con todo su potencial físico ante una situación de emergencia, superar el peligro y lograr un estado de adaptación ante las circunstancias que lo rodean. Esta situación de emergencia o amenaza, conocida como estresor, activa una respuesta neurobiológica de naturaleza protectora, la cual se inicia en el hipotálamo con la liberación de la hormona de corticotropina $(\mathrm{CRH})$ dirigida al sistema circulatorio de la glándula pituitaria, la cual segrega la hormona adenocorticotropa (ACTH) a través del eje hipotálamo-pituitaria-suprarrenal (HPA), produciendo hormonas glucocorticoides, especialmente cortisol, además de catecolaminas como la adrenalina y noradrenalina a través del eje simpático-suprarrenal-medular (SAM) (McEwen, 2007).

Dichas sustancias bioquímicas producen un aumento en la concentración de glucosa en la sangre generando un mayor nivel de energía, oxígeno, alerta, poder muscular y resistencia al dolor en cuestión de minutos (Sapolsky, Romero \& Munck, 2000). La liberación de estas hormonas permite una comunicación inmediata con el sistema nervioso autónomo a través de los sistemas simpático y parasimpático, permitiendo la comunicación del fenómeno de estrés con los aspectos neurobiológicos de la emoción y poner al organismo en estado de alerta, preparándolo para "luchar o huir". Al mismo tiempo, la liberación del cortisol facilita la preparación del sistema inmune para manejar las defensas contra bacterias, virus, heridas e inflamaciones que pudieran ocurrir como resultado del evento estresante. Los mecanismos neurobiológicos de la respuesta del estrés juegan un rol primordial en el 
proceso de adaptación y supervivencia. El incremento de CRH, ACTH, cortisol, adrenalina, noradrenalina, vasopresina y otras hormonas producidas por la glándula pituitaria durante una situación de estrés es de naturaleza protectora y adaptativa con el propósito de mantener el equilibrio alostático (McEwen, 1998).

Esta breve explicación de la respuesta neurobiológica del estrés nos ilustra acerca de la naturaleza benévola y adaptativa de dicho proceso. Cuando la situación de estrés agudo se transforma en crisis repetitivas de distrés emocional generado por el fenómeno de estrés crónico, el cerebro a través del eje hipotálamo-pituitaria-suprarrenal produce un giro de 180 grados en estas mismas funciones neurobiológicas y causa una alteración bioquímica caracterizada por una excesiva utilización, o por el contrario, de un manejo ineficiente de las hormonas indicadas anteriormente y propiciando de esta manera la expresión de un cuadro clínico conceptualizado como distrés emocional percibido (Moscoso, McCreary, Goldenfarb, Knapp, \& Reheiser, 2000; Moscoso, Lengacher \& Reheiser, 2012). La respuesta a esta primera interrogante es el punto de quiebre del desbalance generado por este exceso permanente de demandas ambientales, las que producen un significativo desgaste en las funciones neurobiológicas de los sistemas nerviosos e inmune y ocasionan el inicio del proceso denominado carga alostática (McEwen, 2006). Este giro de 180 grados en las funciones neurobiológicas del organismo, causado por la experiencia de estrés crónico y la expresión del distrés emocional percibido, explica la naturaleza emocional de la primera interrogante planteada en nuestro artículo (Moscoso, 2009).

La segunda cuestión requiere una explicación de naturaleza neurobiológica, en la medida que concierne las funciones de los glucocorticoides y en particular del cortisol en el cerebro y sistema nervioso, y sus efectos negativos sobre el organismo. Los efectos acumulativos del estrés crónico resultan en la alteración de las funciones del cortisol como mediador central de la respuesta neurobiológica del estrés, reflejando un aumento en la producción o una insuficiencia en la liberación de esta hormona (Koob,1999). Este desbalance o resistencia glucocorticoide, originado en las glándulas suprarrenales, produce un significativo desgaste en las funciones del cerebro y sistema inmune, causando serias alteraciones físicas y mentales. El principio central en este desbalance está basado empíricamente en que el estrés crónico mantiene niveles elevados de corticoesteroides en ciertas estructuras cerebrales, como la amígdala y el hipocampo, así como también en las células del sistema inmune (Sapolsky, 1986). Estas elevadas concentraciones de cortisol generan una respuesta de inhibición de los receptores de esta hormona en dichas estructuras cerebrales y células blancas del sistema inmune y producen una estimulación en la liberación de las citoquinas proinflamatorias (Sapolsky, 1996).

En la actualidad existe una sólida base empírica que indica que el cortisol tiene un rol central en la regulación de las respuestas pro y antiinflamatorias, siendo 
este el mecanismo neurobiológico causante de una serie de disfunciones autoinmunes y metabólicas. Las elevadas concentraciones de cortisol producen una crisis energética en las neuronas del cerebro por exceso de glucosa en las células de grasa. Esta acumulación de glucosa en las células de grasa inhibe el $75 \%$ de absorción de glucosa en ciertas estructuras cerebrales y con ello causa una crisis energética en el individuo. Este ejemplo nos ilustra la interacción del estrés crónico y las concentraciones elevadas de cortisol en la fatiga crónica y otras enfermedades metabólicas. Asimismo, la experiencia de distrés emocional presenta un correlato bioquímico en el lóbulo prefrontal del cerebro, lo que indica que la experiencia de estrés crónico se inicia y afecta al cerebro de manera directa, produciendo alteraciones en neuronas de la amígdala y del hipocampo, que resultan en disfunciones de la memoria y aprendizaje (McEwen, 1998; McEwen, 2001, Sapolsky, 1996).

\section{Efectos del estrés crónico sobre las emociones: distrés emocional percibido}

El estrés crónico tiene un impacto negativo muy significativo en las emociones y el comportamiento del individuo. Con el propósito de tener un entendimiento claro sobre las consecuencias emocionales que un individuo experimenta de estresores de la vida diaria, es necesario distinguir operacionalmente el concepto de estrés crónico y las características emocionales relacionadas con este constructo, es decir, los síntomas de distrés emocional que forman parte del concepto erróneamente denominado "estresado" (Moscoso, Lengacher \& Reheiser, 2012). En primer lugar, es importante reconocer que la experiencia de distrés emocional, como consecuencia afectiva y comportamental del estrés crónico, se inicia en el cerebro debido a su rol mediador de la respuesta neurobiológica del estrés.

En segundo lugar, la experiencia de distrés emocional causada por el estrés crónico es un proceso fundamentalmente cognitivo que genera una alteración en la formación de emociones caracterizadas como negativas y/o positivas, con una tendencia al incremento de las emociones negativas y el consecuente desencadenamiento de cuadros de ansiedad, ira y depresión clínica (Spielberger \& Moscoso, 1996; Moscoso, 2011). Este desbalance cognitivo-emocional del individuo cumple un rol desencadenante en una serie de mecanismos de activación neurobiológica en el sistema límbico, y de manera específica en el lóbulo prefrontal, la amígdala y el hipocampo, a los cuales nos hemos referido en líneas anteriores (McEwen, 2007).

El distrés emocional percibido es un cuadro clínico desencadenado por el estrés crónico y su medición representa un tema importante de investigación en neurociencia cognitiva. Desde el punto de vista conceptual, el distrés emocional se define como "un estado marcado por sentimientos que varian en intensidad, desde tristeza, inseguridad, confusión y preocupación, hasta la experiencia de síntomas mucho más severos como por ejemplo la ansiedad, depresión, ira, aislamiento social y pérdida de esperanza" (Moscoso, 2011; Moscoso, McCreary, Goldenfarb, Knapp \& Reheiser, 2000). Nuestra labor empírica en pacientes con 
cáncer nos ha permitido corroborar que el concepto de distrés emocional percibido incluye los síntomas de ansiedad, ira, pérdida de esperanza y depresión, los cuales deben ser claramente distinguidos del diagnóstico de una depresión mayor (Moscoso, Lengacher \& Reheiser, 2012). Asimismo, estudios empíricos realizados por Cassileth, Lusk, Hutter, Strouse \& Brown (1984) sugieren que los síntomas de ansiedad y depresión representan simplemente dos rótulos aparentemente diferentes, con un elevado nivel de correlación entre ambos. Estas afirmaciones indican que la ansiedad y la depresión clínica "marchan de la mano", lo cual confirma nuestras observaciones empíricas en pacientes con cáncer.

\section{Reducción del estrés crónico: mito o realidad}

En párrafos anteriores hemos descrito brevemente los efectos negativos del estrés crónico sobre el cerebro y el sistema inmune, así como sus efectos en las emociones y la expresión de distrés emocional. Estas "cascadas", neurobiológica y comportamental, nos pueden dejar la impresion de que las dificultades de la vida y situaciones de adversidad nos conducen inevitablemente a un "callejón sin salida", en el cual estamos destinados a claudicar frente a la experiencia del estrés y finalmente terminar en un nosocomio o en el cementerio. Si realmente hay algo de cierto en este último párrafo, la acumulación de evidencia científica en el campo de la psicoterapia e intervenciones relacionadas con la reducción de estrés crónico nos demuestran que "al final del túnel” existen suficientes razones para sentir esperanza y optimismo. En este sentido, es el momento de referirnos y reflexionar acerca del desarrollo de una actitud resiliente, que es inherente a la naturaleza humana. Una vez más, es necesario plantear una tercera interrogante empírica: (3) ¿Qué opciones tenemos para prevenir y reducir los efectos nocivos del estrés crónico sobre la salud del individuo?

La tercera cuestión empírica involucra la práctica de un paradigma terapéutico cognitivo, con un significativo énfasis en prevención y cambio de estilo de vida. Este modelo toma en cuenta un cambio actitudinal y comportamental con el propósito de incentivar conductas que expresen un mayor nivel de resiliencia (Moscoso, 2009). El avance científico acerca del entendimiento de los mecanismos neurobiológicos de la respuesta del estrés crónico ha facilitado la implementación de la terapia cognitiva centrada en mindfulness como uno de los modelos de tratamiento más efectivos en neurociencia cognitiva (Segal, Williams \& Teasdale, 2001). Este innovador programa de intervención psicológica pone énfasis en la reducción del distrés emocional, tomando en consideración factores relacionados con el ambiente social, estilos de vida e interacciones neurobiológicas. Dicho programa de entrenamiento cognitivo facilita la respuesta de relajación y la modificación de patrones cognitivos autodestructivos, favoreciendo el desarrollo de emociones positivas (Garland, Gaylord \& Fredrickson, 2011). De igual manera, facilita el desarrollo de un estilo de vida saludable con el propósito de promover la calidad de vida del paciente. 


\section{El estrés crónico en la experiencia del paciente con diagnóstico de cáncer}

El diagnóstico de cáncer y sus tratamientos representan una experiencia sumamente estresante para el paciente y su familia. La expresión de distrés emocional en pacientes con cáncer activa un conjunto de síntomas caracterizados por estados de ansiedad, temor de recurrencia del tumor, insomnio, ira, depresión, y en muchos casos un desorden de estrés postraumático (Lengacher et al., 2009; Moscoso, Reheiser \& Hann, 2004). Asimismo, pacientes con diagnóstico de cáncer experimentan una activación neurobiológica de la respuesta de estrés con un compromiso de los ejes HPA y SAM, los que incluyen disturbios inmunológicos tales como significativas elevaciones y/o reducciones en cortisol, reducción de linfocitos y un aumento de citoquinas proinflamatorias (Lengacher et al., 2012).

Nuestros estudios de investigación en la Universidad de South Florida (USF) están diseñados con el propósito de examinar la eficacia del entrenamiento en reducción de estrés basado en la práctica de mindfulness, específicamente en pacientes con diagnóstico de cáncer. Las metas propuestas en nuestra labor empírica tienen como objetivo observar los cambios cognitivos y neurobiológicos que los pacientes con cáncer presentan en el curso de sus tratamientos a base de quimioterapia, radioterapia o cirugía, como también después de haber finalizado dichos tratamientos (Lengacher et al., 2007).

La metodología empírica utilizada en nuestros protocolos clínicos responde a los criterios del modelo de estrés crónico descrito en el presente estudio, lo cual permite la posibilidad de observar las diferencias clínicas en (1) variables cognitivas como la ansiedad, depresión, temor de recurrencia del tumor y distrés emocional (Lengacher et al., 2009); (2) variables somáticas como la fatiga, dolor e insomnio (Lengacher et al, 2012); y (3) variables neurobiológicas en referencia con los niveles de cortisol (corticoesteroides), linfocitos, citoquinas proinflamatorias y telomerasa (Lengacher et al., 2013; Lengacher et al., 2014; Moscoso, 2014).

Con el propósito de ilustrar la metodología empírica indicada en el párrafo anterior, es conveniente presentar una breve descripción de un estudio piloto de nuestro laboratorio, en el que se examinó los efectos de un programa de reducción de estrés basado en mindfulness (MBSR), basado en el programa original de Jon Kabat-Zinn (Kabat-Zinn, 1990), en relación con factores psicológicos, síntomas físicos, cortisol salivaria y la interleucina-6 (citoquina proinflamatoria) en pacientes con cáncer avanzado y los responsables de sus cuidados (Lengacher et al., 2012).

El objetivo del estudio fue evaluar la viabilidad y el nivel de efectividad del programa MBSR modificado para pacientes con cáncer, observando si, al final del entrenamiento MBSR, los pacientes presentan un nivel de mejoría de sus síntomas psicológicos, físicos y de calidad de vida. Asimismo, nos propusimos examinar los niveles de reducción de estrés crónico tanto en los pacientes como en las personas encargadas del cuidado del paciente (Lengacher et al., 2012).

La investigación fue diseñada como un estudio piloto, por el hecho de ser considerado uno de los primeros trabajos empíricos de naturaleza exploratoria con 
este tipo de pacientes. Se utilizó el modelo cuasiexperimental de un solo grupo con la prueba de pre-post, tipificando al estudio como un ejemplo de modelo traslacional con la idea de examinar el nivel de eficacia del programa MBSR en pacientes con cáncer. A base de la metodología utilizada, los participantes fueron seleccionados utilizando criterios de inclusión de pacientes adultos en estados avanzados de cáncer de mama, próstata, pulmones y colon (estadios III y IV), quienes habían completado sus tratamientos de quimioterapia, radioterapia y/o cirugía. Los pacientes fueron reclutados en el Moffitt Cancer Center de la Universidad de South Florida. Todo el grupo de pacientes del estudio $(n=26)$ y los familiares responsables del cuidado de dichos pacientes $(n=26)$ participaron en nuestro programa MBSR de 6 semanas de duración (Lengacher et al., 2012).

Los resultados del estudio indican que los pacientes presentaron un mejoramiento significativo $(\mathrm{p}<.05)$ después de participar en el programa MBSR de 6 semanas. Dicho mejoramiento se pudo observar en los indicadores de estrés percibido, ansiedad, depresión y síntomas físicos medidos por la Escala Memorial de Medicion de Síntomas (MSAS). En cuanto a los familiares encargados del cuidado del paciente participante del programa, se observó un mejoramiento en los aspectos psicológicos y de calidad de vida, sin embargo dicho mejoramiento no fue estadísticamente significativo. En cuanto a los niveles de cortisol, tanto los pacientes como los familiares responsables del cuidado presentaron notables reducciones después de participar en el programa MBSR (Lengacher et al., 2012).

Al final de la sesión \# 6, las reducciones de cortisol fueron casi la mitad de las medidas observadas antes del inicio del programa, lo cual es notablemente significativo. Asimismo, estas reducciones de cortisol en pacientes y familiares fueron observadas después de la primera sesión de entrenamiento. De manera similar a las reducciones de cortisol, los pacientes y los familiares encargados del cuidado mostraron una reducción notable de citoquinas proinflamatorias al final del programa MBSR, comparado con los niveles de IL-6 presentados antes de iniciar el programa de 6 semanas (Lengacher et al., 2012).

Las conclusiones de nuestro estudio piloto permiten indicar que el programa de reducción de estrés basado en mindfulness es un tipo de intervención beneficiosa para pacientes con diagnóstico de cáncer avanzado y los familiares responsables del cuidado de dichos pacientes. Los pacientes mostraron una significativa reducción de estrés, ansiedad y depresión. La reducción de cortisol y citoquinas proinflamatorias fue también notable en los pacientes y los familiares responsables del cuidado después de recibir el programa de reducción de estrés de 6 semanas (Lengacher et al., 2012).

\section{SUMARIO Y CONCLUSIONES}

El concepto de estrés ha evolucionado significativamente desde los estudios de Cannon y Selye a comienzos del siglo XX. Debido a que este concepto no tiene una valoración definida y carece de especificidad por su naturaleza fenomenológica, el nuevo modelo conceptual de estrés crónico toma en consideración las reacciones 
emocionales y el estilo de vida del individuo. En investigación científica, definimos al estrés crónico como el "resultado de demandas y presiones durante periodos de tiempo prolongados percibidos como eternos o interminables". Este estado emocional es prevalente en pacientes con cáncer, personas expuestas a situaciones de pobreza, en individuos responsables del cuidado prolongado de personas con discapacidad física e individuos que sufren enfermedades crónicas (Moscoso, 2014).

Esta nueva visión del concepto de estrés considera al cerebro como el órgano central de percepción y respuesta a estresores, el cual determina las respuestas neurobiológica y emocional. En este sentido, es de particular importancia el concepto de distrés emocional percibido que permite un mejor entendimiento del impacto de los estresores en la salud del individuo. El estrés crónico ha recibido atención en el campo de la investigación científica debido a su elevada correlación con enfermedades crónicas, como es el caso de las enfermedades autoinmunes, depresión, obesidad, enfermedades cardiovasculares y cáncer, por mencionar algunas. Las alteraciones neurobiológicas causadas por el fenómeno de estrés crónico en los sistemas hormonales reguladores de glucocorticoides y catecolaminas causan un desgaste en ciertas funciones cerebrales e inmunológicas y precipitan estas enfermedades.

Asimismo, el distrés emocional percibido, como resultado de la experiencia de estrés crónico, es tambien un factor importante en la etiología y desarrollo de dichas enfermedades. Este innovador modelo conceptual de investigación científica ignora al concepto de estrés en su forma abstracta y etérea debido a su debilidad metodológica. Desde este nuevo punto de vista, las influencias del cerebro, factores cognitivos y estados emocionales en la salud del individuo toman en consideración el marco conceptual de alostasis y carga alostática, en lugar del inadecuado concepto "estático" de homeostasis (McEwen \& Wingfield, 2003).

El diagnóstico de cáncer representa una experiencia sumamente estresante para el paciente y su familia y describe de manera elocuente el impacto emocional de esta enfermedad en este grupo de personas. La agresividad de los tratamientos oncológicos afecta la calidad de vida del paciente y de los responsables de su cuidado. La expresion de distrés emocional por parte de estas personas activa un conjunto de síntomas caracterizados por estados de ansiedad, temor de recurrencia del tumor, insomnio, ira, depresión, y en muchos casos un desorden de estrés postraumático (Lengacher et al., 2009; Moscoso, Reheiser \& Hann, 2004). Asimismo, pacientes con diagnóstico de cáncer experimentan una activación neurobiológica de la respuesta de estrés con un compromiso de los ejes HPA y SAM, los cuales incluyen disturbios inmunológicos tales como significativas elevaciones y/o reducciones en cortisol, reduccion de linfocitos y un aumento de citoquinas proinflamatorias (Lengacher et al., 2012).

El conocimiento científico de los mecanismos neurobiológicos de la respuesta del estrés crónico ha favorecido el desarrollo de la terapia basada en mindfulness como un programa de tratamiento en neurociencias. Tal como ilustramos en la sección final del presente estudio, las aplicaciones clínicas de este nuevo modelo 
terapéutico han demostrado ser muy efectivas en el contexto del estrés crónico, aliviando la depresión, reduciendo los niveles de distrés emocional y estimulando la creación de estilos de vida más saludables (Lengacher et. al., 2007). Este tipo de intervención psicológica enfatiza la prevención y reducción del distrés emocional dentro del proceso de adaptación al estrés crónico.

\section{REFERENCIAS BIBLIOGRÁFICAS}

Cannon, W.B. (1915). Bodily changes in pain, hunger, fear, and rage. Boston: C.T. Branford Co., Inc.

Cannon, W.B. (1932). The wisdom of the body. New York: W.W. Norton and Co., Inc.

Cassileth, B.R., Lusk, E.J., Hutter, R., Strouse, T.B., \& Brown, L.I. (1984). Concordance of depression and anxiety in patients with cancer. Psychological Reports, 54, 588-590.

Dallman, M.F. (2003). Chronic stress and obesity: A new view of 'comfort food'. Proc. Natl. Acad. Sci. USA; 100:11696-11701.

Dhabhar, F.S., McEwen, B.S. (1997). Acute stress enhances while chronic stress suppresses cell-mediated immunity in vivo: A potential role for leukocyte trafficking. Brain, Behavior, and Immunity. 11(4): 286-306.

Garland, E. L., Gaylord, S. A., \& Fredrickson, B. L. (2011). Positive appraisal mediates the stress-reductive effects of mindfulness: An upward spiral process. Mindfulness, 2, 59-67.

Kabat-Zinn J (1990). Full catastophe living. New York: Delta.

Koob, G.F. (1999). Corticotropin-releasing factor, norepinephrine, and stress. Biol. Psychiat. 46:1167-1180.

Lengacher, C. A., Kip, K. E., Moscoso, M., Johnson-Mallard, V., Molinari, M., Gaurkee, D., et al. (2007). Mindfulness-Based Stress Reduction (MBSR) improves psychological status and general health among breast cancer survivors. Psycho-Oncology 16,3, S72-S73.

Lengacher, CA., Kip, K., Barta, M., Jacobsen, PB., Groer, M., Lehman, B., Moscoso MS., et al. (2012). A pilot study evaluating the effects of Mindfulness Based Stress Reduction on psychological status, physical status, salivary cortisol, Interleukin-6 among advanced-stage cancer patients and their caregivers. Journal of Holistic Nursing. 30, 170-185.

Lengacher, CA., Kip, K., Post-White, J., Fitzgerald, S., Newton, C., Barta., M., Moscoso, MS., et al. (2013 ). Lymphocyte Recovery after Breast Cancer Treatment and Mindfulness-Based Stress Reduction (MBSR) Therapy. Journal of Biological Research for Nursing. 15, 1, 37-47. 
Lengacher, C; Johnson-Mallard, V; Post-White, J; Moscoso, M; Jacobsen, P; Klein, T, et al. (2009). Randomized controlled trial of mindfulness-based stress reduction (MBSR) for survivors of breast cancer. Psycho-Oncology. 18, 12, 1261-1272. Doi: 10.1002/pon.1529: 10.1002/pon.1529

Lengacher, CA., Reich, RR., Kip, KE., Barta, M., Ramesar, S., Paterson, CL., Moscoso, MS., et al. (2014). Influence of Mindfulness-Based Stress Reduction on Telomerase Activity in Women with Breast Cancer (BC). Biological Reseach for Nursing. Doi: 10. 1177.

Lengacher, C; Reich, R; Post-White, J; Moscoso, M; Shelton, M; Barta, M., et al. (2012). Mindfulness based stress reduction in post-treatment breast cancer patients: An examination of symptoms and symptom clusters. Journal of Behavioral Medicine. 35, 86-94. Doi: 2011.10.1007/s10865011-9346-4: 10.1007/s10865-011-9346-4.

Lupien SJ , McEwen BS, Gunnar MR , Heim C (2009). Effects of the stress throughout the lifespan on the brain, behavior and cognition. Nature Reviews Neuroscience, 10, 6, 434-445.

MacPherson, A., Dinkel, K., Sapolsky, R. (2005). Glucorticoids worsen excitotoxin-induced expression of pro-inflammatory cytokines in hippocampal cultures. Exper. Neurol. 194: 376-383.

McEwen, B.S. (1998). Protective and Damaging Effects of Stress Mediators. New England J. Med. 338:171-179.

McEwen, B.S. (2001). Plasticity of the hippocampus: adaptation to chronic stress and allostatic load. Annals of the New York Academy of Sciences. 933: 265-277.

McEwen, B.S. (2006). Sleep deprivation as a neurobiologic and physiologic stressor, allostastis and allostatic load. Metabolism. 55; S20-S23.

McEwen, B.S. (2007). The physiology and neurobiology of stress and adaptation, Central role of the brain. Physiol. Rev. 87:873-904.

McEwen, B.S. \& Stellar, E. (1993). Stress and the individual: Mechanisms leading to disease. Archives of Internal Medicine. 153, 2093-2101.

McEwen, B.S., Wingfield, J.C. (2003). The concept of allostasis in biology and biomedicine. Horm. \& Behav. 43:2-15.

Moscoso, M.S. (1995). Manejo cognitivo-conductual del estres en individuos VIH-1 seropositivos. Avances en Psicologia Clinica Latinoamericana. 13: 39-53.

Moscoso, MS. (2009). De la mente a la celula: Impacto del estres en psiconeuroinmunoendocrinología. Revista Liberabit. 15, 2, 143-152. 
Moscoso, MS. (2010). El estrés crónico y la terapia cognitiva centrada en mindfulness: Una nueva dimension en psiconeuroinmunología. Revista Persona. 13, 11-29.

Moscoso, MS. (2011). El estrés crónico y la medición psicométrica de distrés emocional percibido en medicina y psicología clínica de la salud. Revista Liberabit. 17, 1, 67-76.

Moscoso, MS (2014). El estudio científico del estrés crónico en neurociencias y psicooncología. Revista Persona. 17, 1, 53-70.

Moscoso, MS., Lengacher, CA. \& Reheiser, EC. (2012 ). The assessment of the perceived emotional distress: The neglected side of cancer care. Journal of Psicooncologia. 9, 2-3. 277-288.

Moscoso, M.S., McCreary, D., Goldenfarb, P., Knapp, M., Reheiser, E. (2000). A brief screening inventory to measure emotional distress in cancer patients. Psycho-Oncology, 9(5): S53.

Moscoso, M.S., Reheiser, E.C., Hann, D. (2004). Effects of a brief mindfulness-based stress reduction intervention on cancer patients. Psycho-Oncology. 13(1): S12.

Sapolsky, R.M. (1986). Glucocorticoid toxicity in the hippocampus reversal by supplementation with brain fuels. The Journal of Neuroscience. 6, 22402244.

Sapolsky, R.M. (1996). Why stress is bad for your brain. Science. 273, 749-750.

Sapolsky, R.M., Romero, L.M., Munck, A.U. (2000). How do glucocorticoids influence stress responses? Integrating permissive, suppressive, stimulatory, and preparative actions. Endocrine Rev. 21:55-89.

Selye, H. (1936). A syndrome produced by diverse nocuous agents. Nature. 138: 32 .

Segal, Z.V., Williams, M.G. \& Teasdale, J.D. (2001). Mindfulness-based cognitive therapy for depression: A new approach to preventing relapse. The Guilford Press. Columbus, OH.

Schulkin, J. (2003). Rethinking homeostasis: Allostatic regulation in physiology and pathophysiology. Cambridge, MA. MIT Press.

Spielberger, C.D., Moscoso, M.S. (1996). Reacciones emocionales del estrés: ansiedad y cólera. Avances en Psicología Clínica Latinoamericana. 14: 59-81.

Sterling, P., Eyer, J. (1988). Allostasis, A New Paradigm to Explain Arousal Pathology. In: S. Fisher; J. Reason, (Eds.) Handbook of Life Stress, Cognition and Health. New York: John Wiley \& Sons; p.629-649.

Taub, D. (2008). Novel Connections between the Neuroendocrine and Immune Systems: The Ghrelin Immunoregulatory Network. Vitam. Hor. 77:325-346. 